\title{
Mass SARS-CoV-2 molecular and serological screening of medical staff and patients in Hangzhou, China: no evidence of RNA detection, low seroprevalence, and limited exposure risk in the hospital setting
}

\author{
Jun Huang ${ }^{1 \#}$, Lu Zhang ${ }^{1 \#}$, Shengjun Wu ${ }^{1}$, Jie Lu ${ }^{1}$, Fengying Li ${ }^{1}$, Yulan Cheng ${ }^{1}$, Qi Zhang ${ }^{1}$, Guiling Li ${ }^{1}$, \\ Haitao Yu ${ }^{1}$, Zhian Le ${ }^{1}$, Xiaowen He ${ }^{1}$, Yanjun Ding ${ }^{2}$, Daniel Candotti ${ }^{3}$, Xinyou Xie ${ }^{1}$, Jun Zhang, \\ ${ }^{1}$ Department of Clinical Laboratory, Sir Run Run Shaw Hospital, Zhejiang University School of Medicine, Hangzhou, China; ${ }^{2}$ Key Laboratory of \\ Biotherapy of Zhejiang Province, Hangzhou, China; ${ }^{3}$ National Institute of Blood Transfusion/INTS, Department of Blood Borne Agents, National \\ Reference Center for Infectious Risks in Blood Transfusion, Paris, France \\ Contributions: (I) Conception and design: J Huang, L Zhang, X Xie, J Zhang; (II) Administrative support: X Xie, J Zhang; (III) Provision of study \\ materials or patients: J Huang, S Wu, J Lu, F Li, Y Cheng, Q Zhang, G Li, H Yu, Z Le, X He; (IV) Collection and assembly of data: J Huang, L \\ Zhang, Y Ding; (V) Data analysis and interpretation: J Huang, L Zhang, J Lu, D Candotti; (VI) Manuscript writing: All authors; (VII) Final approval \\ of manuscript: All authors. \\ \#These authors contributed equally to this work. \\ Correspondence to: Jun Zhang, MD; Xinyou Xie, MD. Department of Clinical Laboratory, Sir Run Run Shaw Hospital, Zhejiang University School of \\ Medicine, 3 East Qingchun Road, Hangzhou, China. Email: jameszhang2000@zju.edu.cn; scottxie@zju.edu.cn.
}

Background: To assess and limit the SARS-CoV-2 exposure risk from symptomless individuals in the hospital setting, molecular and serological screening of staff and patients attending a tertiary hospital in China was conducted.

Methods: SARS-CoV-2 RNA was tested by quantitative RT-PCR. Anti-SARS-CoV-2 IgM and IgG were screened initially with two lateral flow immunoassays (LFIs) and further confirmed with three chemiluminescence immunoassays (CLIAs). The assay performance was assessed using archived samples from 32 confirmed COVID-19 cases and 80 healthy individuals.

Results: Between April 24 and May 8, 2020, 16,043 subjects (7,392 medical staff, 4,714 inpatients, 1,209 chaperones, 1,705 outpatients, and 1,023 fever clinic patients) were screened. No subject tested positive for viral RNA. Seventy-three (0.46\%) tested positive for IgM or IgG on the initial LFI screening, of whom 63 were investigated with CLIAs: $2(0.01 \%)$ were confirmed as seroreactive and $18(0.11 \%)$ were indeterminate. Unconfirmed seroreactivity was significantly more frequent in fever clinic patients. The CLIAs showed similar (95.0-100\%) IgM or IgG specificity but higher IgG sensitivity (93.75-96.88\% vs. 31.25-81.25\%) than the LFIs. The confirmed seropositive cases included a previously discharged COVID-19 patient and an undiagnosed symptomless patient showing detectable IgM and IgG over 35 days of follow-up. No transmission was evidenced within the corresponding family cluster.

Conclusions: Low SARS-CoV-2 prevalence and limited exposure risk were observed. Seroprevalence varied between $0.012 \%$ and $0.12 \%$ according to the testing algorithm and the confirmation criteria used, indicating that quality standards for serological tests are needed. Protective immunity in asymptomatic COVID-19 patients who recovered needs to be investigated further, but the associated risk of transmission appeared limited.

Keywords: Mass screening; SARS-CoV-2; nucleic acid test; antibody; seroprevalence

Submitted Oct 28, 2020. Accepted for publication Jan 18, 2021.

doi: 10.21037/atm-20-7163

View this article at: http://dx.doi.org/10.21037/atm-20-7163

(c) Annals of Translational Medicine. All rights reserved. 


\section{Introduction}

The outbreak of severe acute respiratory syndrome coronavirus 2 (SARS-CoV-2) has led to more than 13 million infections and more than 580,000 deaths, as of July 16, 2020. Symptom-based control and prevention of SARS-CoV-2 infection appear inefficient, as shown by a growing number of studies that reported COVID-19 outbreaks in different congregate living situations due to the transmission of asymptomatic or presymptomatic SARS-CoV-2 infections (1-7). Medical institutions are densely populated and consequently at high risk of COVID-19 outbreaks. Arons et al. (2) reported a rapid COVID-19 outbreak in a skilled nursing facility with $56 \%$ of residents with positive test results being asymptomatic at the time of testing. The relatively high proportion of asymptomatic cases has also been documented in women admitted for delivery $(13.5 \%)$ or in patients attending essential orthopedic surgery $(12.1 \%)(4,8)$. Although many studies have reported the clinical characteristics of hospitalized COVID-19 cases, data about the prevalence of SARS-CoV-2 infection, and especially anti-SARS$\mathrm{CoV}-2$ seroprevalence in particular hospital populations, remain poorly investigated. To prevent asymptomatic transmission and ensure a safe medical environment, the National Commission of the People's Republic of China recommended SARS-CoV-2 mass testing of different populations in medical institutions, including patients who need emergency hospitalization, patients attending fever clinics, suspected COVID-19 cases and their close contacts, and medical and technical hospital staff.

Most studies performed mass screening by using highly sensitive and specific nucleic acid testing (NAT) assays to detect SARS-CoV-2 infection $(2,9,10)$. However, NAT has some limitations, including not only cost- and infrastructure-related issues but also possible false-negative results, as suggested by the discrepancy observed among viral RNA testing, serology, and clinical symptoms in some cases (11). A study reported that $7.69 \%$ of suspected COVID-19 cases and $4.3 \%$ of their close contacts with anti-SARS-CoV-2 IgM or IgG reactive tested viral RNAnegative in at least two sequential samples (12). Another study showed that the combination of viral RNA and antibody detection significantly improved the sensitivity of COVID-19 diagnosis, even in the early phase of one week after the onset of symptoms (13).

Aiming to prevent SARS-CoV-2 transmission in a particular hospital setting, Sir Run Run Shaw Hospital,
Zhejiang University School of Medicine, Hangzhou, China, investigated the prevalence of SARS-CoV-2 exposure by molecular and serological mass screening of hospital staff, patients attending fever clinics, inpatients and their chaperones, and outpatients from April to May 2020. We present the following article in accordance with the MDAR reporting checklist (available at http://dx.doi.org/10.21037/ atm-20-7163).

\section{Methods}

\section{Study design}

People attending Sir Run Run Shaw Hospital were tested for SARS-CoV-2 RNA by real-time RT-PCR. In parallel, the proportion of SARS-CoV-2 antibody-positive (IgM or $\mathrm{IgG}$ ) individuals were estimated by using two rapid lateral flow immunoassays (LFIs) simultaneously. Samples that were $\operatorname{IgM}$ or $\operatorname{IgG}$ reactive on at least one assay were considered initially reactive (IR). Subsequently, IR samples were tested with three independent chemiluminescence immunoassays (CLIAs) to confirm the serological status. IR samples that tested IgM or IgG reactive with at least two positive CLIAs were classified as confirmed seroreactive.

\section{Study participants and sample collection}

Nasopharyngeal swabs/sputum and serum samples were collected from hospital staff, inpatients, inpatient chaperones, outpatients, and fever clinic patients of Sir Run Run Shaw Hospital between April and May 2020. Samples with insufficient volume were excluded. In addition, 32 archived serum samples from confirmed COVID-19 patients and 80 negative samples were used as controls to evaluate the analytical performance of the serological assays in the study. The 32 patients were diagnosed with COVID-19 based on SARS-CoV-2 RNA positive testing following symptom onset between January 31 and February 20, 2020. These patients originated from a prison transmission cluster. The first confirmed case was a prison guard who had been to Wuhan between January 14 and January 19, 2020. We collected the blood of confirmed COVID-19 patients on April 27, more than 2 months after their infections were diagnosed. Eighty negative samples were collected from healthy people and confirmed by a negative SARS-CoV-2 RNA test and CT scan in April 2020 at the medical center. All samples were stored at $-20^{\circ} \mathrm{C}$.

According to the Diagnosis and Treatment Protocol 
Table 1 Combinations of antigens used in different SARS-CoV-2 antibody detection assays

\begin{tabular}{lcccc}
\hline \multirow{2}{*}{ Assays } & Type & \multicolumn{3}{c}{ Antigens to detect } \\
& & IgM & lgG & Total Ab \\
\hline Livzon (LFI-1) & LFI & rNP-S1-RBD & rNP-S1 & NA \\
Vazyme (LFI-2) & LFI & rNP-S2 & rNP-S2 & NA \\
YHLO (CLIA-1) & CLIA & rNP-S1 & rNP-S1 & NA \\
Wantai (CLIA-2) & CLIA & RBD & rNP & RBD \\
Bioscience (CLIA-3) & CLIA & RBD & RBD & NA \\
\hline
\end{tabular}

rS1, recombinant S1 subunit of SARS-CoV-2 spike protein; rNP-S2, nucleoprotein and spike protein S2 subunit recombinant; rNP-S1, nucleoprotein and spike protein S1 subunit recombinant; $\mathrm{RBD}$, spike protein receptor binding domain; rNP, recombinant nucleoprotein; NA, not applicable.

for COVID-19 (Trial Version 7) published by the National Health Commission of the People's Republic of China, a case was suspected when presenting with any epidemiological history or at least two clinical manifestations in the absence of clear epidemiological history (14).

\section{SARS-CoV-2 NAT}

SARS-CoV-2 RNA was detected in nasopharyngeal swabs or sputum by using a quantitative real-time RT-PCR (Biogerm, Shanghai, China) targeting both the $\mathrm{N}$ gene and ORF1ab gene according to the manufacturer's instructions. Forward and reverse primers and probes for the $\mathrm{N}$ gene were 5'-GGGGAACTTCTCCTGCTAGAAT-3', 5'-CAGACATTTTGCTCTCAAGCTG-3', and 5'-VICTTGCTGCTGCTTGACAGATT-VIC-3', respectively. Forward and reverse primers and probes for the ORF1ab gene were 5'-CCCTGTGGGTTTTACACTTAA-3', 5'-ACGATTGTGCATCAGCTGA-3', and 5'-FAMCCGTCTGCGGTATGTGGAAAGGTTATGG-FAM-3', respectively. The $95 \%$ limit of detection (LoD) was 1,000 copies/mL for both amplification reactions.

\section{Serological testing}

Serological mass screening was performed using two LFIs: LFI-1 (Livzon, Zhuhai, Guangzhou, China) and LFI-2 (Vazyme, Nanjing, Jiangsu, China), simultaneously. Samples IR with LFI were tested further with three CLIAs: CLIA-1 (YHLO, Shenzhen, Guangdong, China), CLIA-2 (Wantai,
Beijing, China) and CLIA-3 (Bioscience, Tianjin, China). The LFIs and CLIAs used different antigen recombinants including the S1 subunit (rS1), nucleoprotein (rNP), and spike protein receptor-binding domain (RBD) of the SARS$\mathrm{CoV}-2$ spike protein to detect IgM and IgG (Table 1).

\section{Statistical analysis}

Statistical analyses were performed using the SPSS package, v21 (SPSS Inc., Chicago, IL, USA). Descriptive statistics, such as medians with ranges and proportions were calculated for most variables. Statistical comparisons between groups were evaluated using the chi-square test. A $P$ value less than 0.05 was considered significant.

\section{Ethical statement}

The study conformed to the provisions of the Declaration of Helsinki (as revised in 2013), available at: https://www. wma.net/wp-content/uploads/2016/11/DoH-Oct2013JAMA.pdf. This study was approved by the Medical Ethical Committee of Sir Run Run Shaw Hospital (Scientific Research 20200331-45). As data were anonymous, written informed consent was waived for the archived specimens used. Written informed consent was obtained only from the subjects who were found to have evidence of active or past SARS-CoV-2 infection, and their close contacts for additional investigations.

\section{Results}

\section{Mass screening for SARS-CoV-2 exposure}

From April 24, 2020 to May 8, 2020, a total of 16,043 subjects were included in the study. The characteristics of the included populations were described in Table 2. None of these subjects tested SARS-CoV-2 RNA reactive by realtime RT-qPCR (Figure 1). Serological screening with LFIs showed no IgM or IgG reactivity in 15,970 samples (99.54\%). IgM and IgG were detected by at least one assay in $50(0.31 \%)$ and $26(0.16 \%)$ samples, respectively. Overall, the concordance between LFI-1 and LFI-2 testing results was $99.85 \%(16,019 / 16,043)$ and $99.86 \%(16,021 / 16,043)$ for IgM and IgG, respectively. However, only $52.0 \%(26 / 50)$ of IgM+ and $15.4 \%(4 / 26)$ of $\mathrm{IgG}+$ samples were reactive consistently with the two assays (Figure 1). Overall, 73 $(0.46 \%)$ samples in which IgM or IgG was IR with at least one assay included $3 \mathrm{IgM+/IgG+}, 23 \mathrm{IgM}-/ \mathrm{IgG}+$, and 47 
Table 2 Characteristics of included populations

\begin{tabular}{lc}
\hline Parameters & $\mathrm{N}(\%)$ \\
\hline Age (years), mean \pm SD & $44 \pm 16$ \\
Gender & $16,043(100.0)$ \\
Male & $6,556(40.9)$ \\
Female & $9,487(59.1)$ \\
Population & $16,043(100.0)$ \\
Medical staff & $7,392(46.1)$ \\
Inpatients & $4,714(29.4)$ \\
Patient chaperones & $1,209(7.5)$ \\
Outpatients & $1,705(10.6)$ \\
Fever clinic patients & $1,023(6.4)$ \\
\hline
\end{tabular}

IgM+/IgG-.

Sixty-three samples that were IR for anti-SARS-CoV-2 (42 IgM+/IgG-, $18 \mathrm{IgM}-/ \mathrm{IgG}+$, and $3 \mathrm{IgM+} / \mathrm{IgG}+$ ) were tested with three CLIA assays for confirmation. Ten IR samples could not be investigated further due to sample volume limitations. Two samples were confirmed to be consistently seroreactive with the three CLIA assays (Table 3). Patient 1 was IgM and IgG IR with the two LFI assays and confirmed as IgM CLIA reactive with optical density/ cutoff (S/CO) ratios of 1.37 (CLIA-1), 17.97 (CLIA-2), and 88.14 (CLIA-3), and IgG reactive with S/CO ratios of 7.87 (CLIA-1), 8.7 (CLIA-2), and 23.17 (CLIA-3). Patient 2 was IgG IR with LFI-2 and only confirmed as IgG reactive with S/CO values of 10.04 (CLIA-1), 16.73 (CLIA-2), and 36.65 (CLIA-3). In addition, 14 out of 45 (31.1\%) IgM IR samples that were reactive with only one CLIA assay were considered not confirmed (Table 3). Ten LFI-1+/LFI-2+ and three LFI-1+/LFI-2- samples were CLIA-1-only reactive [median S/CO: 2.31 (range, 1.06-6.32)] and one LFI-1+/ LFI-2+ sample was CLIA-3-only reactive (S/CO: 6.22). Similarly, six out of 21 (28.7\%) IgG IR samples (1 LFI-1+/ LFI-2+ and 5 LFI-1+/LFI-2-) were reactive with CLIA1 only [median S/CO: 1.49 (range, 1.03-1.98)]. Overall, 18 subjects were considered to have an indeterminate serological status. The distribution of the 61 unconfirmed LFI IR samples in the different populations was analyzed (Table 4). A significantly higher rate of unconfirmed LFI reactivity was observed in the group of patients attending the fever clinic $[1.2 \%(12 / 1,023)]$ than in the other four groups. The lowest IR rate was observed among medical staff $(0.2 \%, 13 / 7,392)$. Unconfirmed LFI reactivity was essentially associated with $\operatorname{IgM}$ positive results $[65.6 \%$ (40/61)] irrespective of the population studied. Overall, the serology confirmation rate was $3.2 \%(2 / 63)$, which led to an estimated seroprevalence of $0.01 \%(2 / 16,033)$ in the population studied.

\section{Comparison of serological assay performance in SARS- CoV-2 RNA-positive and SARS-CoV-2 RNA-negative populations}

The analytical performance of LFI and CLIA assays was evaluated using two control groups that included 32 confirmed SARS-CoV-2 RNA positive patients (infected controls) and 80 SARS-CoV-2 RNA negative and symptomless individuals (non-infected controls). The concordance between LFI-1 and LFI-2 in RNA+ samples was $87.5 \%(28 / 32)$ and $50.0 \%(16 / 32)$ for $\operatorname{IgM}$ and $\operatorname{IgG}$, respectively. As shown in Table 5, the LFI-1 assay had higher $\operatorname{IgM}$ and $\operatorname{IgG}$ detection rates than the LFI-2 assay [IgM: $18.75 \%(6 / 32)$ versus $6.25 \%(2 / 32), \mathrm{P}=0.13$; IgG: $81.25 \%$ (26/32) versus $31.25 \%$ (10/32), $\mathrm{P}<0.001]$. CLIA3 had a significantly higher detection rate $[65.63 \%(21 / 32)]$ than CLIA-1 [18.75\% (6/32)] and CLIA-2 [18.75\% (6/32)] which had detection rates similar to LFI-1 $[18.75 \%(6 / 32)]$. In contrast, all CLIA assays showed similar IgG detection rates $(93.75-96.88 \%)$ that were significantly higher than those obtained with LFI assays (31.25-81.25\%). The median S/CO ratios observed in IgM and IgG CLIA reactive samples were 2.35 and 4.60 with CLIA-1, 1.86 and 9.9 with CLIA-2, and 1.73 and 13.35 with CLIA-3, respectively. All samples that tested IgM reactive on at least one assay were found to also be IgG reactive, except for one sample in which IgM had low reactivity with CLIA-3 (S/ CO: 1.46). All IgG positive samples were reactive with the three CLIA assays, except for one sample with low reactivity with CLIA-2 (S/CO: 1.21) but IgM reactivity with CLIA3 (S/CO: 3.73). Combining IgM and IgG detection seemed to have no improvement on the sensitivity of serological screening except for CLIA-3 (Table 5).

In the negative control, LFI assays showed 100\% specificity for both IgM and IgG (Table 5). Similarly, CLIA1 and CLIA-3 showed $100 \%$ specificity for IgM and IgG, respectively. IgM specificity was $98.75 \%$ and $97.5 \%$ for CLIA-2 and CLIA-3, and IgG specificity was $98.75 \%$ and 95.0\% for CLIA-1 and CLIA-2, respectively. Irrespective of the assay used, S/CO values in reactive samples were borderline suggesting false positivity. Overall, the nature and number of viral antigens used in the different 

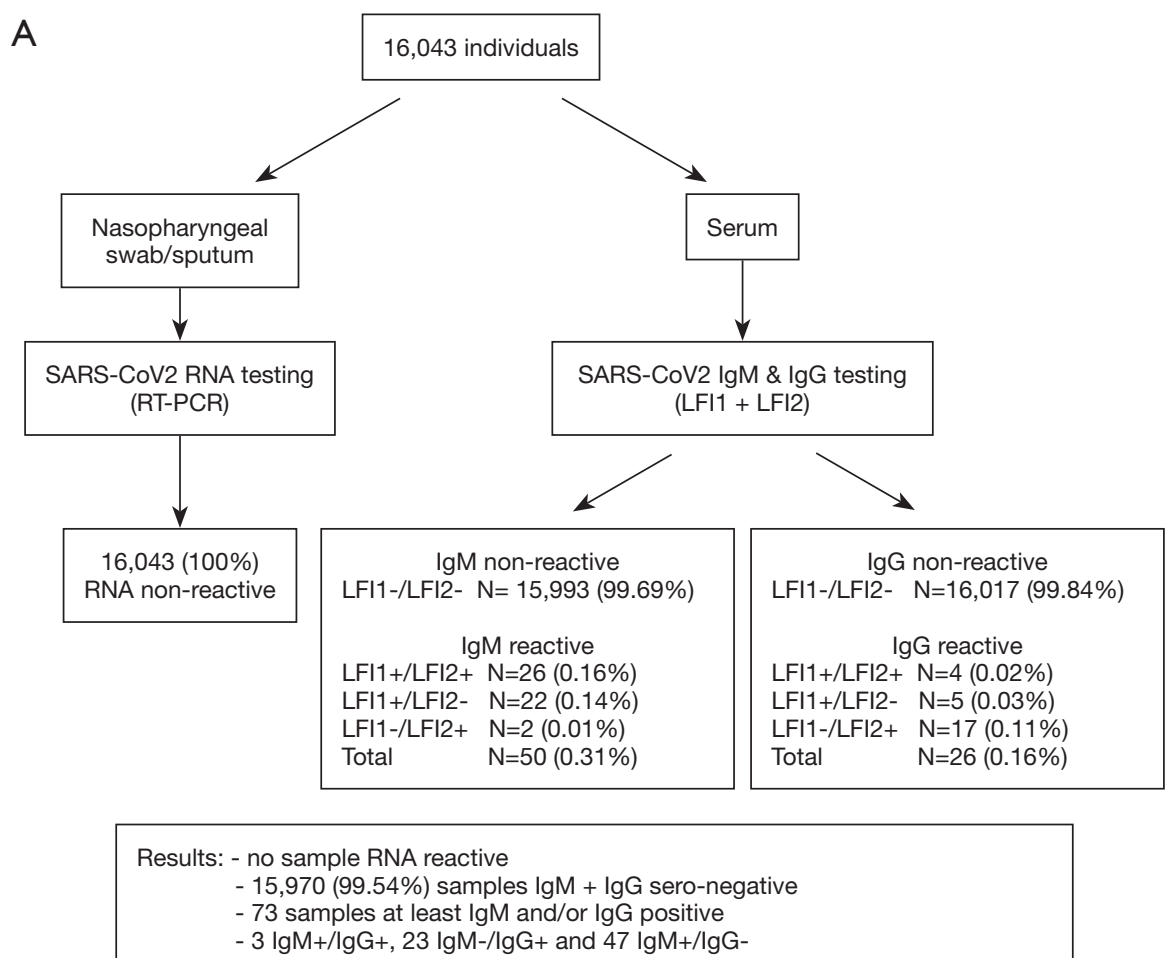

B Confirmatory antibody testing of $63 \mathrm{IgM}$ and/or IgG LFI-reactive samples with 3 CLIA assays
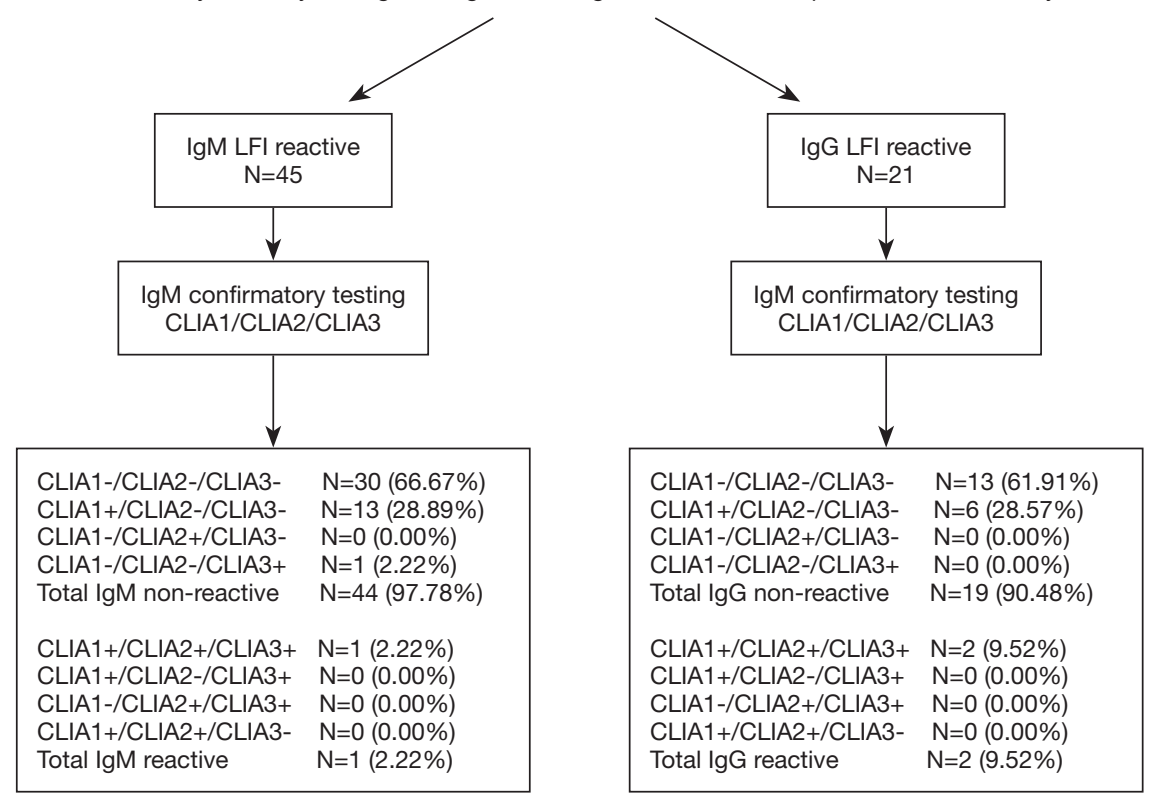

Final serology results

$\begin{array}{ll}\text { lgM-/lgG- } & \mathrm{N}=61(96.82 \%) \\ \text { lgM+/lgG+ } & \mathrm{N}=1(1.59 \%) \\ \text { lgM-/lgG+ } & \mathrm{N}=1(1.59 \%) \\ \text { lgM+/lgG- } & \mathrm{N}=0(0.00 \%) \\ \text { Total sero+ } & \mathrm{N}=2(3.16 \%)\end{array}$

Figure 1 Summary of the (A) mass screening and (B) confirmation results. 
Table 3 Concordance between LFI and CLIA testing results

\begin{tabular}{|c|c|c|c|c|}
\hline CLIA results & Total & $\begin{array}{l}\mathrm{LFI}-1+/ \\
\mathrm{LFI}-2+\end{array}$ & $\begin{array}{l}\text { LFI-1+/ } \\
\text { LFI-2- }\end{array}$ & $\begin{array}{l}\text { LFI-1-/ } \\
\text { LFI-2+ }\end{array}$ \\
\hline $\begin{array}{l}\text { Total IgM non-confirmed } \\
\text { reactive }\end{array}$ & 44 & 22 & 20 & 2 \\
\hline CLIA-1-/CLIA-2-/CLIA-3- & 30 & 11 & 17 & 2 \\
\hline CLIA-1+/CLIA-2-/CLIA-3- & 13 & $10^{\mathrm{a}}$ & $3^{b}$ & 0 \\
\hline CLIA-1-/CLIA-2+/CLIA-3- & 0 & 0 & 0 & 0 \\
\hline CLIA-1-/CLIA-2-/CLIA-3+ & 1 & $1^{\mathrm{c}}$ & 0 & 0 \\
\hline Total IgM confirmed reactive & 1 & 1 & 0 & 0 \\
\hline CLIA-1+/CLIA-2+/CLIA-3+ & 1 & $1^{d}$ & 0 & 0 \\
\hline CLIA-1+/CLIA-2-/CLIA-3+ & 0 & 0 & 0 & 0 \\
\hline CLIA-1-/CLIA-2+/CLIA-3+ & 0 & 0 & 0 & 0 \\
\hline CLIA-1+/CLIA-2+/CLIA-3- & 0 & 0 & 0 & 0 \\
\hline $\begin{array}{l}\text { Total lgG non-confirmed } \\
\text { reactive }\end{array}$ & 19 & 2 & 3 & 14 \\
\hline CLIA-1-/CLIA-2-/CLIA-3- & 13 & 1 & 3 & 9 \\
\hline CLIA-1+/CLIA-2-/CLIA-3- & 6 & $1^{\mathrm{e}}$ & 0 & $5^{\dagger}$ \\
\hline CLIA-1-/CLIA-2+/CLIA-3- & 0 & 0 & 0 & 0 \\
\hline CLIA-1-/CLIA-2-/CLIA-3+ & 0 & 0 & 0 & 0 \\
\hline Total IgG confirmed reactive & 2 & 1 & 0 & 1 \\
\hline CLIA-1+/CLIA-2+/CLIA-3+ & 2 & $1^{g}$ & 0 & $1^{\text {h }}$ \\
\hline CLIA-1+/CLIA-2-/CLIA-3+ & 0 & 0 & 0 & 0 \\
\hline CLIA-1-/CLIA-2+/CLIA-3+ & 0 & 0 & 0 & 0 \\
\hline CLIA-1+/CLIA-2+/CLIA-3- & 0 & 0 & 0 & 0 \\
\hline
\end{tabular}

a, S/CO values of the ten samples were $2.31,6.32,1.97,1.13$, $3.79,3.61,1.06,2.26,1.96$, and $2.43 ;^{b}, \mathrm{~S} / \mathrm{CO}$ values of the three samples were $7.52,1.06$, and 6.07 ; $^{\mathrm{c}}, \mathrm{S} / \mathrm{CO}$ value was 6.22 ; ${ }^{\mathrm{d}}, \mathrm{S} /$ $\mathrm{CO}$ values were $1.37,17.97$, and 88.14 with the three assays, respectively; ${ }^{e}, \mathrm{~S} / \mathrm{CO}$ value was $1.09 ;{ }^{\dagger}, \mathrm{S} / \mathrm{CO}$ values of the five samples were $1.98,1.74,1.74,1.23$, and $1.03 ;{ }^{9}, \mathrm{~S} / \mathrm{CO}$ values were $7.87,8.7$, and 23.17 with the three assays, respectively; ${ }^{\mathrm{h}}, \mathrm{S} / \mathrm{CO}$ values were $10.04,16.73$, and 36.65 with the three assays, respectively. LFI, rapid lateral flow immunoassay; CLIA, chemiluminescence immunoassay.

serological assays seemed to have no significant impact on antibody detection efficiency.

\section{Characterization of SARS-CoV-2 infected patients}

Patient 1 was a 54-year female who visited the hospital because of gallbladder discomfort on May 6, 2020. Routine laboratory tests including lymphocyte counts and high-sensitivity C-reactive protein (hs-CRP) were normal. The CT image showed ground-glass opacities on the middle lobe of right lung, as previously reported on August 7, 2019. She had no typical lower respiratory symptoms, such as fever, cough, and shortness of breath, but transient diarrhea in March, 2020. She was followedup one month later (June 10, 2020). CBC, hs-CRP, and routine chemical and coagulation markers were within the normal reference range (data not shown). She tested negative for SARS-CoV-2 RNA and was confirmed IgM and IgG reactive with the three CLIA assays in May and June. According to direct S/CO values and plasma limiting dilution testing results with the three CLIA assays, anti-SARS-CoV-2 IgM remained detectable at slightly decreasing levels between May and June (Figure 2). The antibody titers measured with CLIA-2 and CLIA-3 using RBD as the target antigen were higher than the titer measured with CLIA-1 that used an NP-S1 recombinant antigen. In contrast, anti-SARS-CoV-2 IgG levels seemed to slightly increase over time as suggested in Figure 2. A consistent 1:160 titer of anti-RBD total antibodies was measured with the CLIA-2 assay over the follow-up period (data not shown). Patient 1 reported no contact with confirmed or suspected COVID-19 patients, and she had not travelled outside Hangzhou since December 2019. In addition, close relatives including her husband, daughter and son, who had lived with her since December 2019 were tested and followed-up. Both NAT and antibody testing remained negative for the three family members over time suggesting an absence of SARS-CoV-2 transmission within this family cluster.

Patient 2 was a previously discharged COVID-19 patient who visited the hospital for follow-up testing. She was a 38-year female and first visited the hospital because of chest distress on January 31, 2020. She reported a history of contact with relatives from Wuhan, China on January 25 and January 28, 2020. She had a little cough and sputum with CT images showing pulmonary inflammation. She was identified as confirmed case on February 7, 2020 based on the positive NAT result from the Hangzhou Center for Disease Control and Prevention. On April 30, Patient 2 tested SARS-CoV-2 RNA negative, IgM-/IgG+. IgG S/ CO ratios of $10.78,14.62$ and 13.15 and dilution titers of 1:20, 1:40 and 1:2 were obtained with CLIA-1, CLIA-2, and CLIA-3, respectively. S/CO ratio of 410.09 and 1:160 
Table 4 Distribution of 61 unconfirmed LFI initially reactive samples in different populations

\begin{tabular}{|c|c|c|c|c|c|}
\hline Population & $\mathrm{N}$ subjects & LFI+/CLIA- (\%) & $\operatorname{lgM}+/ \operatorname{lgG}-(\%)$ & $\operatorname{lgM}-/ \lg G+(\%)$ & $\operatorname{lgM}+/ \lg G+(\%)$ \\
\hline Inpatients & 4,714 & $26(0.55)$ & $14(0.29)$ & $11(0.23)$ & $1(0.02)$ \\
\hline Patient chaperones & 1,209 & $5(0.41)$ & $5(0.41)$ & 0 & 0 \\
\hline Outpatients & 1,705 & $5(0.29)$ & $4(0.23)$ & $1(0.06)$ & 0 \\
\hline Total & 16,043 & $61(0.38)$ & $40(0.25)$ & $20(0.12)$ & $1(0.01)$ \\
\hline
\end{tabular}

${ }^{a}, \mathrm{P}<0.001$ compared with medical staff group; ${ }^{\mathrm{b}}, \mathrm{P}=0.02$ compared with inpatients group; ${ }^{\mathrm{c}}, \mathrm{P}=0.04$ compared with patient chaperones group; ${ }^{d}, P=0.02$ compared with outpatients group. LFI, lateral flow immunoassay.

Table 5 Analytical performance of different SARS-CoV-2 antibody detection assays

\begin{tabular}{|c|c|c|c|c|c|c|c|}
\hline Assays & Antibody type & \multicolumn{3}{|c|}{ RNA+ samples (N=32) } & \multicolumn{3}{|c|}{ RNA- samples ( $\mathrm{N}=80$ ) } \\
\hline \multirow{2}{*}{ LFI-1 } & $\lg G$ & $26 / 32$ & 81.25 (62.96-92.14) & NA & $0 / 80$ & 100 (94.29-100) & NA \\
\hline & $\operatorname{lgM} / \lg G$ & $26 / 32$ & 81.25 (62.96-92.14) & NA & $0 / 80$ & 100 (94.29-100) & NA \\
\hline \multirow{2}{*}{ LFI-2 } & $\lg G$ & $10 / 32$ & $31.25(16.75-50.14)$ & NA & $0 / 80$ & 100 (94.29-100) & NA \\
\hline & $\operatorname{lgM} / \lg G$ & $10 / 32$ & $31.25(16.75-50.14)$ & NA & $0 / 80$ & 100 (94.29-100) & NA \\
\hline CLIA-1 & $\lg M$ & $6 / 32$ & $18.75(7.86-37.04)$ & $2.35(1.03-12.42)$ & $0 / 80$ & 100 (94.29-100) & NA \\
\hline \multirow{3}{*}{ CLIA-2 } & $\lg G$ & $31 / 32$ & 96.88 (82.00-99.84) & $9.9(1.21-17.34)$ & $4 / 80$ & 95.00 (87.01-98.39) & $1.16(1.05-1.24)$ \\
\hline & $\operatorname{lgM} / \lg G$ & $31 / 32$ & 96.88 (82.00-99.84) & NA & $5 / 80$ & 93.75 (85.38-97.68) & NA \\
\hline & Total Ab & $30 / 32$ & 93.75 & $66.51(6.3-393.3)$ & $0 / 80$ & & \\
\hline \multirow[t]{3}{*}{ CLIA-3 } & $\lg M$ & $21 / 32$ & 65.63 (46.77-80.83) & $1.73(1.0-136.9)$ & $2 / 80$ & $97.50(90.43-99.57)$ & $2.69(2.08-3.29)$ \\
\hline & $\lg G$ & $30 / 32$ & 93.75 (77.78-98.91) & $13.35(1.66-88.1)$ & $0 / 80$ & $100(94.29-100)$ & NA \\
\hline & $\operatorname{lgM} / \lg G$ & $32 / 32$ & $100(86.66-100)$ & NA & $2 / 80$ & $97.50(90.43-99.57)$ & NA \\
\hline
\end{tabular}

titer of anti-RBD total antibodies were measured with the CLIA-2 assay (data not shown).

\section{Discussion}

In the present study, mass testing of SARS-CoV-2 nucleic acid and specific antibodies in 16,043 subjects attending Sir
Run Run Shaw Hospital, Zhejiang University, Hangzhou, China, resulted in no subject testing positive for SARSCoV-2 RNA, and 73 subjects initially IgM or IgG reactive with LFI of which only $2(0.01 \%)$ were confirmed with CLIA.

Similar results have been reported from other cities in China. For example, in Wuhan, the epicenter of the 

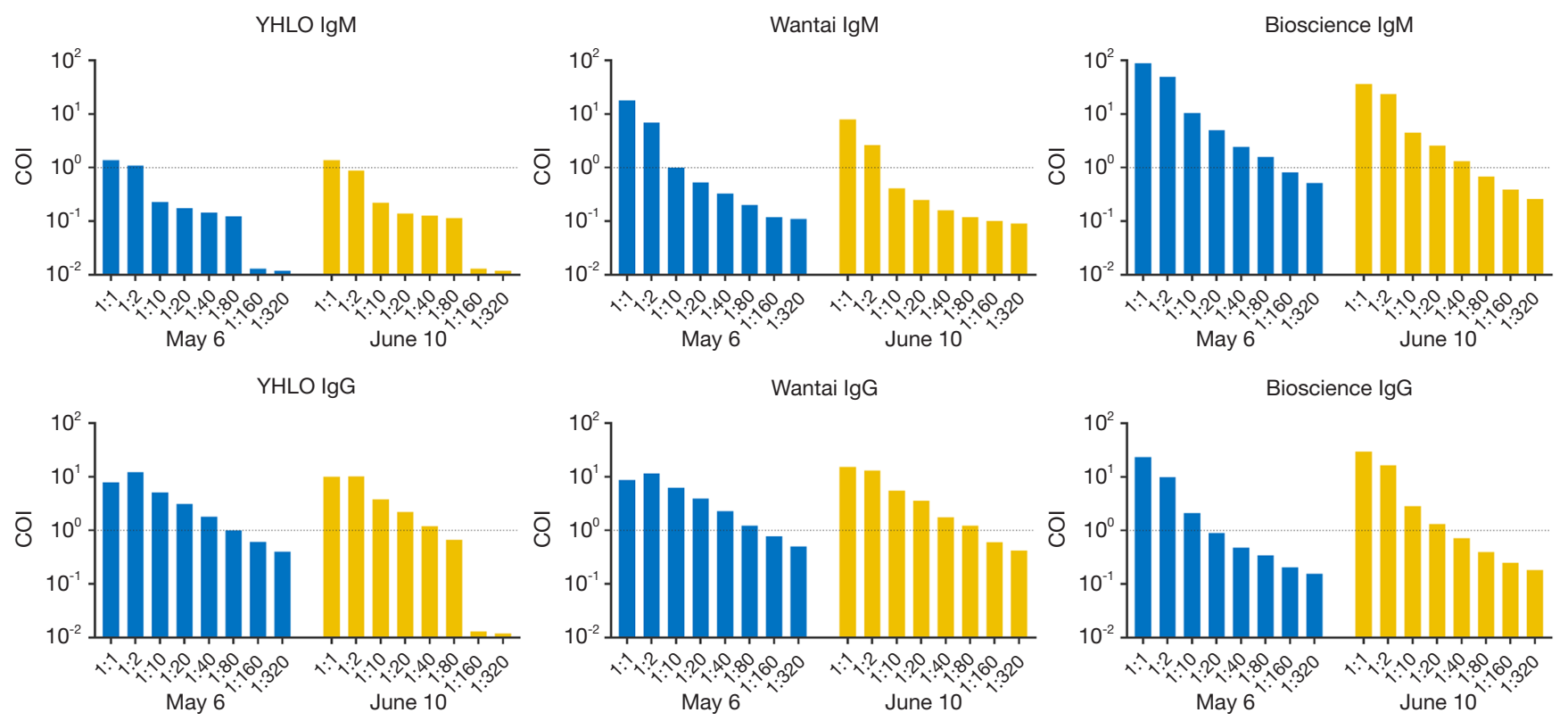

Figure 2 Antibody titers of Patient 1 using three CLIAs during follow up. CLIAs, chemiluminescence immunoassays.

epidemic, 189 (0.03\%o) asymptomatic viral RNA carriers were identified out of 6.574 million people tested between May 14 and 23, 2020 (15). However, studies from other countries reported much higher RNA positive rates ranging from $3 \%$ to $19 \%$ in asymptomatic healthcare workers, residents and staff members in long-term care skilled nursing facilities, and women admitted for delivery $(4,16,17)$. The molecular testing of upper or lower respiratory tract samples by RT-PCR remains the gold standard despite several limitations that include long turnaround times and up to $30 \%$ false negatives due to technical errors and sampling time (18).

Antibody tests are likely to have a complementary role in detecting ongoing and previous SARS-CoV-2 infections $(19,20)$. However, the duration and level of antibody production are still uncertain, and seroprevalence surveys of large populations have been somewhat limited to date. A study reported a seroprevalence of $10.8 \%$ in Geneva, Switzerland, which was significantly higher than that in the present study (16). In contrast, a recent study from Guangzhou, China, reported a $0.09 \%$ seroprevalence of SARS-CoV-2 IgG and IgA in asymptomatic blood donors with no viral RNA reactivity (21). Establishing the accurate seroprevalence of SARS-CoV-2 may prove particularly challenging. Although a wide range of tests are now available, their analytical performance is often poorly documented. In the present study, the performance of the serological assays used was evaluated by using confirmed RT-PCR-positive and RT-PCR-negative samples as controls according to the World Health Organization (WHO) and China National Health Commission of the People's Republic of China (CDC) case definitions. However, there were several limitations that included the relatively limited numbers of available samples in both control groups, and, more importantly, uncertainties about the SARS-CoV-2 serological status of infected and non-infected controls. Indeed, potential recovery from past infection with persistently detectable antibodies could not be completely ruled out in RNA negative controls. Nevertheless, based on the results obtained in the RNA negative control group, the specificity of both the LFI and CLIA assays appeared to be satisfactory (95.0-100\%). The weak and occasional IgM or IgG reactivity (S/CO $<1.5$ with CLIA- 1 and CLIA2 assays, and $<3.5$ with CLIA-3) observed in few samples suggested that the likelihood of false positivity was higher than past exposure to the virus. Sensitivity was evaluated in RNA positive patients more than two months after the onset of symptoms and molecular diagnosis. A previous study showed that SARS-CoV-2 specific IgG was detectable at day 12 after the onset of symptoms in patients with severe and mild COVID-19 disease, and then rapidly and continuously increased within the next 6 weeks. In contrast, SARS-CoV-2 specific IgM was detected on day 9, persisted at a high level for approximately two weeks, and then 
decreased to the threshold detection limit within the next 6 weeks, which suggested that SARS-CoV-2 specific IgM remained measurable for a shorter period than $\operatorname{IgG}(20)$. This might explain the difference between IgM and IgG sensitivity observed when testing control samples irrespective of the serological assay used (Table 5). In addition, Zeng et al. suggested that severe COVID-19 cases generally had earlier IgM responses and higher IgM and IgG levels against SARS-CoV-2 than mild cases. Hence, it remains unclear whether the assays used in the present study could be sensitive enough to detect low antibody levels, which were likely to be associated with asymptomatic SARS$\mathrm{CoV}-2$ infection. SARS-CoV-2 seroprevalence may be underestimated in the population studied, as the initial screening was performed using LFI assays that showed limited sensitivity compared to CLIA assays. Furthermore, only samples reactive with at least one LFI assay and confirmed with two CLIA assays were classified as true positives for anti- SARS-CoV-2, while additional 18 samples were considered serologically indeterminate despite being $\operatorname{IgM}$ or IgG reactive with at least one LFI assay and one CLIA assay. CLIA S/CO ratios were on the low side but with no significant difference as compared to IgM or IgG S/CO values measured in RNA positive controls [IgM median $\mathrm{S} /$ CO: 2.31 (1.06-7.52) vs. 2.35 (1.03-12.42), $\mathrm{P}=0.58$; IgG: 1.49 (1.03-1.98) vs. 4.42 (1.01-10.55), $\mathrm{P}=0.23$ ]. In addition, with LFI-1, LFI-2, and CLIA-1 showing IgM or IgG specificity of $98.75-100 \%$ (Table 5), the likelihood of false positivity was low. Therefore, if true seropositivity cannot be totally excluded in these indeterminate samples, the estimated SARS-CoV-2 seroprevalence might be $0.12 \%$ $(20 / 16,033)$. On the other hand, 43 additional samples were found to be $\operatorname{IgM}(n=30)$ or $\operatorname{IgG}(n=13)$ reactive with LFI assays but not with CLIAs, which suggested initial false positivity. However, these discordant results contradicted the sensitivity and specificity observed in the different assays (Table 5). In addition, unconfirmed IR samples were more often found in patients attending the fever clinic $(1.17 \%$ vs. $0.18-0.55 \% ; \mathrm{P}<0.04$; Table 4$)$, suggesting potential unspecific cross-reactivity, unrelated to SARS-CoV-2 infection, associated with the immunological and infection status of these particular patients. These discrepancies need to be investigated further. Meanwhile, these differences between subjects and controls testing highlighted the need for the determination of appropriate cutoffs per assay type.

The low prevalence of SARS-CoV-2 infections observed in our study is consistent with the prevalence situation in Hangzhou during which there was no new case reported (22).
However, there was few data available about mass screening in the community and the comparison can't be performed, which was a limitation in this study. Overall, the low prevalence of SARS-CoV-2 infection observed in our study may reflect the epidemic situation in Hangzhou, which may be attributed to the effective application of control measures and the well-controlled epidemic situation in Hangzhou, Zhejiang Province, China. Zhejiang Province initiated the most restrictive social distancing and quarantining measures at a very early stage, and it was the first city in China to apply digital mobile tracking, which contributed to rapidly decreasing the net reproduction number (Rt). In addition, individuals suspected of having COVID-19 symptoms were systematically referred to specifically dedicated hospitals, which likely contributed to the low prevalence observed in Sir Run Run Shaw Hospital. However, the similarity of data obtained in the population of a general hospital in Hangzhou to that in blood donors in Guangzhou suggested a relatively limited exposure of the general population to the virus. It has been estimated that the herd immunity threshold for SARS-CoV-2 is approximately $67 \%$ when the net Rt is approximately 3 (23). Hence, the sometimes proposed strategy to establish herd immunity through natural exposure seems limited, and a mass vaccination program may be considered a more valuable option to develop immunity against this virus.

Patient 2 was a discharged COVID-19 patient who visited the hospital for follow-up 88 days after symptom onset and presented an IgM negative/IgG positive serological profile characteristic of a recovered viral infection. Patient 1 reported no typical COVID-19 symptoms since December 2019, and SARS-CoV-2 infection was unsuspected before entering this mass screening study. Overall molecular and serological data suggested that Patient 1 had been infected with SARSCoV-2 shortly before May 6, 2020, and recovered from the infection. No specific route of infection could be definitively documented. However, she had worked in her own store since March 2020, which may have constituted a risk of exposure to the virus. Interestingly, she did not transmit the virus to any of the family members living with her during this period, in contrast to several previous reports about cluster transmission induced by asymptomatic cases $(6,7)$, or by COVID-19 outbreaks in congregate living situations (1). The absence of transmission may be related to the undetectable RNA documented in this patient, or to the presence of potential neutralizing antibodies. Patient 1 had relatively high and stable titers of anti-NP IgG (1:80), 
over time but lower titers of anti-RBD IgG (1:10 in May and 1:20 in June). This was in agreement with other studies reporting a high level of anti-NP IgG among recovered COVID-19 patients ( $\geq 1: 320)$ (24). However, increasing evidence has shown that antibodies targeting RBD have potent neutralizing activity against SARS-CoV-2 (25-29), while protective immunity associated with anti-NP IgG remains poorly documented. Further studies are still needed to establish whether apparently recovered patients, such as patient 1 , benefit from long-term protective immunity, and what level of protection is conferred by the different types of antibodies naturally produced.

In conclusion, molecular and serological mass screening for SARS-CoV-2 revealed an extremely low prevalence of exposure to the virus in individuals attending Sir Run Run Shaw Hospital in Hangzhou. SARS-CoV-2 seroprevalence varied from $0.012 \%$ to $0.12 \%$ according to the testing algorithm and confirmation criteria used. The establishment of quality standards for SARS-CoV-2 serological tests is critical. The level of protective immunity in asymptomatic COVID-19 patients who recovered remained uncertain, but the associated risk of transmission appeared limited.

\section{Acknowledgments}

Funding: Medical and Health Science and Technology Project of Zhejiang Province 2020KY158; Department of Education, Zhejiang Province Y202043596; Medical and Health Science and Technology Project of Zhejiang Province 2017KY418.

\section{Footnote}

Reporting Checklist: The authors have completed the MDAR reporting checklist. Available at http://dx.doi.org/10.21037/ atm-20-7163

Data Sharing Statement: Available at http://dx.doi. org/10.21037/atm-20-7163

Conflicts of Interest: All authors have completed the ICMJE uniform disclosure form (available at http://dx.doi. org/10.21037/atm-20-7163). The authors have no conflicts of interest to declare.

Ethical Statement: The authors are accountable for all aspects of the work in ensuring that questions related to the accuracy or integrity of any part of the work are appropriately investigated and resolved. The study was conducted in accordance with the Declaration of Helsinki (as revised in 2013), available at: https://www.wma.net/wpcontent/uploads/2016/11/DoH-Oct2013-JAMA.pdf. This study was approved by the Medical Ethical Committee of Sir Run Run Shaw hospital (Scientific Research 20200331-45). As data were anonymous, written informed consent was waived for the archived specimens used. Written informed consent was obtained only from the subjects who were found with evidence of active or past SARSCoV-2 infection, and their close contacts for additional investigations.

Open Access Statement: This is an Open Access article distributed in accordance with the Creative Commons Attribution-NonCommercial-NoDerivs 4.0 International License (CC BY-NC-ND 4.0), which permits the noncommercial replication and distribution of the article with the strict proviso that no changes or edits are made and the original work is properly cited (including links to both the formal publication through the relevant DOI and the license). See: https://creativecommons.org/licenses/by-nc-nd/4.0/.

\section{References}

1. Gandhi M, Yokoe DS, Havlir DV. Asymptomatic transmission, the Achilles' heel of current strategies to control COVID-19. N Engl J Med 2020;382:2158-60.

2. Arons MM, Hatfield KM, Reddy SC, et al. Presymptomatic SARS-CoV-2 infections and transmission in a skilled nursing facility. N Engl J Med 2020;382:2081-90.

3. Mayor S. Covid-19: Nine in 10 pregnant women with infection when admitted for delivery are asymptomatic, small study finds. BMJ 2020;369:m1485.

4. Sutton D, Fuchs K, D'Alton M, et al. Universal screening for SARS-CoV-2 in women admitted for delivery. N Engl J Med 2020;382:2163-4.

5. Wei WE, Li Z, Chiew CJ, et al. Presymptomatic transmission of SARS-CoV-2 - Singapore, January 23-March 16, 2020. MMWR Morb Mortal Wkly Rep 2020;69:411-5.

6. Day M. COVID-19: identifying and isolating asymptomatic people helped eliminate virus in Italian village. BMJ 2020;368:m1165.

7. Bai Y, Yao L, Wei T, et al. Presumed asymptomatic carrier transmission of COVID-19. JAMA 2020;323:1406-7.

8. Gruskay JA, Dvorzhinskiy A, Konnaris MA, et al. Universal testing for COVID-19 in essential orthopaedic 
surgery reveals a high percentage of asymptomatic infections. J Bone Joint Surg Am 2020;102:1379-88.

9. Pan X, Chen D, Xia Y, et al. Asymptomatic cases in a family cluster with SARS-CoV-2 infection. Lancet Infect Dis 2020;20:410-1.

10. Peto J, Alwan NA, Godfrey KM, et al. Universal weekly testing as the UK COVID-19 lockdown exit strategy. Lancet 2020;395:1420-1.

11. Caruana G, Croxatto A, Coste AT, et al. Diagnostic strategies for SARS-CoV-2 infection and interpretation of microbiological results. Clin Microbiol Infect 2020;26:1178-82.

12. Long QX, Liu BZ, Deng HJ, et al. Antibody responses to SARS-CoV-2 in patients with COVID-19. Nature Medicine 2020;26:845-8.

13. Zhao J, Yuan Q, Wang H, et al. Antibody responses to SARS-CoV-2 in patients of novel coronavirus disease 2019. Clin Infect Dis 2020;71:2027-34.

14. National Health Commission of the people's Republic of China. the Diagnosis and Treatment Protocol for COVID-19 (2020 Trial Version 7). Available online: http:// wwwnhcgovcn/yzygj/s7653p/202003/46c9294a7dfe4cef80 dc7f5912eb1989shtml

15. Wuhan Government. Coronavirus disease 2019 (COVID-19): situation report. Available online: http:// wjw.wuhan.gov.cn/ztzl_28/fk/yqtb/

16. Stringhini S, Wisniak A, Piumatti G, et al. Seroprevalence of anti-SARS-CoV-2 IgG antibodies in Geneva, Switzerland (SEROCoV-POP): a population-based study. Lancet 2020;396:313-9.

17. Rivett L, Sridhar S, Sparkes D, et al. Screening of healthcare workers for SARS-CoV-2 highlights the role of asymptomatic carriage in COVID-19 transmission. eLife 2020;9:e58728.

18. Xiao AT, Tong YX, Zhang S. False negative of RT-PCR

Cite this article as: Huang J, Zhang L, Wu S, Lu J, Li F, Cheng Y, Zhang Q, Li G, Yu H, Le Z, He X, Ding Y, Candotti D, Xie X, Zhang J. Mass SARS-CoV-2 molecular and serological screening of medical staff and patients in Hangzhou, China: no evidence of RNA detection, low seroprevalence, and limited exposure risk in the hospital setting. Ann Transl Med 2021;9(7):552. doi: 10.21037/atm-20-7163 and prolonged nucleic acid conversion in COVID-19:

Rather than recurrence. J Med Virol 2020;92:1755-6.

19. Sethuraman N, Jeremiah SS, Ryo A. Interpreting Diagnostic Tests for SARS-CoV-2. JAMA 2020;323:2249-51.

20. Zeng Z, Chen L, Pan Y, et al. Re: Profile of specific antibodies to SARS-CoV-2: The first report. J Infect 2020;81:e80-e81.

21. Xu R, Huang J, Duan C, et al. Low prevalence of antibodies against SARS-CoV-2 among voluntary blood donors in Guangzhou, China. J Med Virol 2021;93:1743-7.

22. Hangzhou Municipal Health Commission. Coronavirus disease (COVID-19) outbreak situation. Available online: http://wsjkw.hangzhou.gov.cn/col/ col1229008279/index.html

23. Randolph HE, Barreiro LB. Herd Immunity: Understanding COVID-19. Immunity 2020;52:737-41.

24. Zhang L, Pang R, Xue $X$, et al. Anti-SARS-CoV-2 virus antibody levels in convalescent plasma of six donors who have recovered from COVID-19. Aging (Albany NY) 2020;12:6536-42.

25. Gao Q, Bao L, Mao H, et al. Development of an inactivated vaccine candidate for SARS-CoV-2. Science 2020;369:77-81.

26. Wu Y, Wang F, Shen C, et al. A noncompeting pair of human neutralizing antibodies block COVID-19 virus binding to its receptor ACE2. Science 2020;368:1274-8.

27. Pinto D, Park YJ, Beltramello M, et al. Crossneutralization ofSARS-CoV-2 by a human monoclonal SARS-CoV antibody. Nature 2020;583:290-5.

28. Ju B, Zhang Q, Ge J, et al. Human neutralizing antibodies elicited by SARS-CoV-2 infection. Nature 2020;584:115-9.

29. Wrapp D, De Vlieger D, Corbett KS, et al. Structural basis for potent neutralization of betacoronaviruses by single-domain camelid antibodies. Cell 2020;181:1004-15. 